\title{
Lymph Node Sinus
}

National Cancer Institute

\section{Source}

National Cancer Institute. Lymph Node Sinus. NCI Thesaurus. Code C33033.

An irregular tortuous space within lymph nodes through which a continuous stream of lymph passes entering into the efferent lymphatic vessels. 